\title{
An Innovative Method of Estimation Hewing for Invention Report Mining and Estimation Summarization
}

\author{
S. Ravichandran ${ }^{1}$ and J. Sathiamoorthy ${ }^{2}$ \\ ${ }^{1}$ Professor in Computer Science Department, Annai Fathima College of Arts \& Science, Madurai, Tamil Nadu, India \\ ${ }^{2}$ Associate Professor in Department of Software Applications, Thiruthangal Nadar College, Chennai, Tamil Nadu, India \\ E-mail: drravichandran6@gmail.com, jsathyam74@gmail.com
}

\begin{abstract}
With the assistance of Web 2.0, the bases on client interest, posting on the web surveys has become an undeniably mainstream path for individuals to impart their perspectives to different client's suppositions and conclusions toward items and administrations. It turns into a typical practice for online business sites to give the offices to individuals to convey and distribute their audits between them. These online audits present an abundance of data on the Services and Products, which will encourage the improvement of their business. Consequently a developing number of late examinations have been centred on the Opinion Mining. For example the Opinion Mining alludes to computational method for assessing the sentiments that are mined from different Web Sources.
\end{abstract}

A couple of Opinion Mining based techniques have been considered and broke down. From our investigation, it is seen that a couple of feeling mining based directed and unaided techniques had not delivered great outcomes because of alluding less number of sentiments inside a similar URL'S and treating the highlights with comparable significance as various. To beat this issue, Topic Anatomy Model TSCAN was proposed, where the Task is called as Topic Anatomy and which sums up and relates the primary pieces of a point with the goal that the per users could comprehend the substance without any problem.

By utilizing this model, the more data can be removed and related through their transient closeness, which will give conceivable substance. This model is including imperative part in the Opinion Mining since clients can impart their insights about the items. From our usage, it is seen that this plan gives the best reasonable answer for the client's advantages and requests. Notwithstanding, it burns-through more opportunity to anticipate the best performing items because of huge informational collections respectively.

Consequently our exploration work is proposed and actualized a productive strategy for Opinion Mining called an Efficient Parallel Opinion Mining (EPOM) constructed TSCAN Algorithm separately. It is centring more sites and it is removing more data in equal way, so we can get advanced productive outcome with least execution time. From our outcomes, it is noticed that it gives the best reasonable answer for the client's advantages and requests and it $I \mathrm{~s}$ improving the presentation of existing method regarding Quality of Information, Prediction and Execution Time. Keywords: Text Mining, Sentiment classification, summarization, URL, reviews

\section{INTRODUCTION}

Prior to the Web, when an individual expected to settle on a choice, he/she regularly requested feelings from loved ones. At the point when an association needed to discover the suppositions or assessments of the overall population about its items and administrations, it gathered information, overviews, and centre gatherings. As a rule, suppositions are covered up in long discussion posts and sites. It is hard for a human per user to discover pertinent sources, extricate related sentences with suppositions, understood them, sum up them, and coordinate them into usable structures. Consequently, computerized assessment revelation and synopsis frameworks are required. Estimation examination, otherwise called an assessment mining, outgrows this need. Sentiment mining is the idea under the Data mining, where it is a subsequent strategy for extricating, grouping, discerning and evaluating the assessments verbally expressed in the various sites, web-based media inner parts and other client produced setting.

The audit of client typically incorporates the item assessments of a great deal of clients articulated in an assortment of structures along with regular language sentences. In for the most part individuals generally don't offer their thoughts in straightforwardly. For Ex., a portion of the items may have the highlights like "the focal point in the camera is acceptable and the focal point sets aside too long effort for centring the article" The fundamental intension of the assessment mining is to foresee the feelings for the items and highlights of those items from the different web assets. Past examinations on sentiment mining have applied different strategies for highlight extraction and refinement, including NLP and measurable techniques. Nonetheless, these examinations uncovered the accompanying issues. It doesn't centres around the more sites for alluding the sentiment. It prompts helpless irregularity of the information. The assessments are anticipated with in similar URLs. The clients who are completely posted the remarks in numerous URLs will prompts best assessment of the sentiment expectation. Rather than anticipating the conclusions in a similar URL, alluding numerous URLs and preparing the feelings in those URLs will give best appropriate answer for the clients [2]. To determine these issues, this paper proposes an improved 
strategy called, proficient equal Opinion Mining based Texamine Based Algorithm (EPOM).

The general cycle of EPOM comprises of three stages: web assortment data, assessment direction cycle, and production of representation instrument. In Web assortment data the way toward investigating the message will be happen. To acquire this the necessary information will be given. After this cycle the assessment direction will happens. Here the way toward extricating the suppositions and assessment types are concluded. At that point with the perception device the necessary diagram configuration will be acquired.

\section{RELATED WORK}

Mining $\mathrm{Hu}$ [1] considered as the pioneer work to discover the synopsis dependent on element and assessment. They have utilized affiliation rule mining to discover successive thing sets, gotten from each sentence thing phrases. To prune the continuous things they have utilized various strategies. The rare highlights are distinguished dependent on the assessment word present in the sentence. Outline comprising of the item include and the sentiment on it has been given regarding positive and negative.

G. Carenini [8] proposed a methodology for mining item highlight and sentiment dependent on the thought of syntactic and semantic data. They have utilized reliance relations and ontological information with probabilistic model. They have additionally utilized Product Ontology to recognize comparable component with various wording.

Yuanbin Wu et al [5] developed their own reliance parser, to recognize the item includes and the assessment on these highlights from the item audits. The feeling related with the element is distinguished dependent on the window size of 5 from the separated word to the sentiment word present in that sentence.

Parma Nand [6] proposed a calculation for settling anaphora dependent on notability loads. He has zeroed in on settling anaphors especially in the class of in short paper type articles since it frames a piece of more extensive exploration pointed toward building a framework for perception of online paper articles. The created calculation is equipped for settling the anaphors utilizing information helpless methodology which is totally founded on remarkable quality scores.

Chih-Ping Wei et al. [7] utilized the methodology, to mine item highlights and feeling on these highlights utilizing the semantic based methodology. By and by the methodology depends on co event of thing phrase and the feeling word.

Wong TL [3] utilized syntactic pieces of information got from reliance parse tree as highlights for foreseeing the power of feeling phrases.

\section{III.PROBLEM STATEMENT}

This is the design chart for the current framework and it consummately works and appropriate for the anticipating the assessments in a similar URL. Also, it neglects to anticipate the sentiments dependent on many URL's.

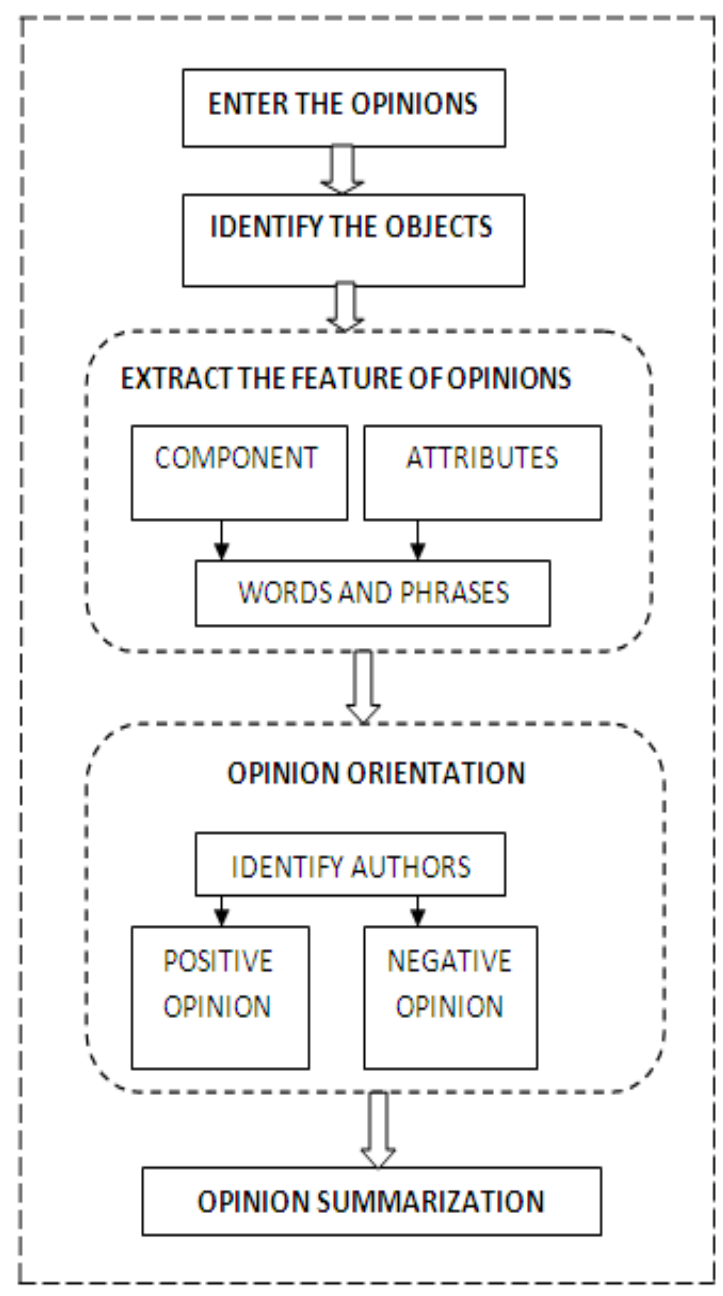

Fig.1 Common Architecture Design

The means included are as follow:

1. Feature extraction and equivalent word gathering

2. Opinion direction assurance

3. Integration

In light of the over four factors the cycle has happens in the current method. In this framework the conclusions are anticipated inside similar URLs. It doesn't canters different URLs and it prompts helpless distinguishing proof of the conclusions. The clients who are completely posted the remarks in the numerous URLs will prompt best assessment of the feeling forecast. Rather than anticipating the sentiments in a similar URL, eluding numerous URLs and handling the assessments in those URLs will give best reasonable answer for the clients. The reference of single URL's will prompts the in proficient alluding of the sentiments and precision of the data is extremely low [3]. In 
this EPOM the above issues will be cleared by suing the TSCAN calculation. So the exactness of data and more assessments are eluded in the proposed framework. No other existing papers which depend on the assessment mining don't zero in on the numerous URLs. Furthermore, this task work simply dependent on numerous URL's which will give reasonable answers for the clients.

\section{IMPLEMENTATION}

This work is wanted to plan a proficient model called Efficient Parallel Opinion Mining (EPOM), it will give best appropriate answer for the client's advantages and requests.

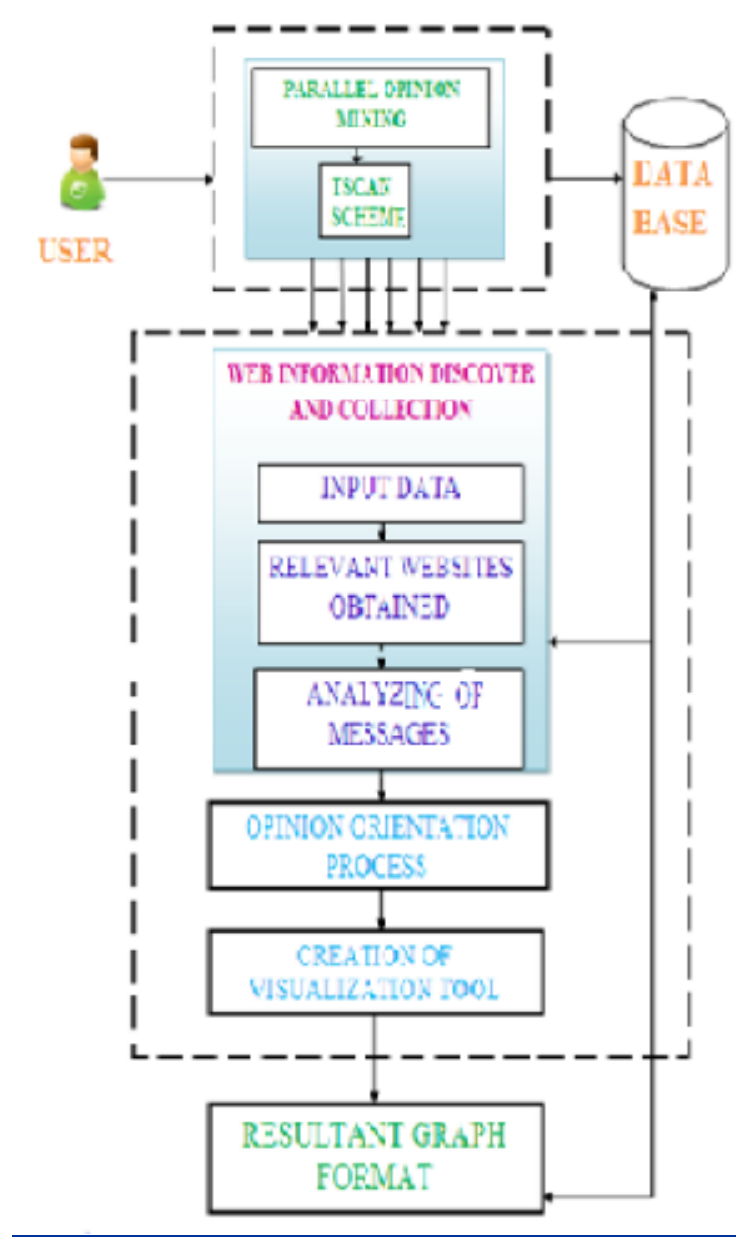

Fig.2 Implementing Architecture Design

For example this proposed model is centring more sites and it will extricate more data in equal way, so we can get enhanced outcome with least execution time [6]. Accordingly, this proposed work will improve the exhibition of existing procedure regarding Quality of data, Prediction and execution time.

This is the precise design graph for the proposed framework. Here with the assistance of equal feeling mining and TSCAN plot the cycle has happens. The following stage to this is web data find and assortment. Here the info information is taken first after that the applicable sites are done. The investigating of messages will do after the getting of the significant sites. In the assessment direction measure the feeling characters will be investigated. The perception apparatus is utilized for making the resultant chart design which will be helpful organization to the clients. The acquired data's can store in the information base for the future reference.

\section{A. Algorithm Used}

The procedure utilized here in this EPOM is TSCAN calculation. The proposed point life structures model, called TSCAN, determines the significant subjects of a theme from the eigenvectors of a worldly square affiliation network respectively. By this point, the huge occasions of the topics and their outlines are extricated by analysing the constitution of the eigenvectors. The proposed TSCAN disintegrates each report into a succession of noncovering blocks. A square can be a few successive sentences, or at least one sections. We characterize a square as $\mathrm{w}$ continuous sentences. The Theme is produced by developing square affiliation grid because of extricating the passages with an enormous worth infers a high relationship between the comparing pair of squares. In light of the Theme produced, it successfully mine the web assessments synopsis [1]. This proposed work will improve the exhibition of existing strategy as far as Quality of data, Prediction and execution time. There are three models used.

\section{Topic Model}

In This theme model it considers the remarks as squares/sentences and the squares are further splatted for the occasion arrangement.

A subject is a true episode that involves at least one subjects, which are identified with a better occurrence, a depiction, or a discourse about a specific issue. During the life expectancy of a subject, one topic may pull in more consideration than the others, and is along these lines detailed by more reports. We characterize an occasion as a critical topic improvement that proceeds for a while. Normally, all the occasions taken together structure the storyline(s) of the point. In spite of the fact that the occasions of a subject are transiently disjoint, they are viewed as semantically subordinate to communicate the advancement of the topic. In addition, occasions in various subjects might be related due to their worldly vicinity and setting likeness [8]. The proposed strategy distinguishes subjects and occasions from the point's reports, and interfaces related occasions to frame the theme's advancement diagram. Moreover, the recognized occasions are summed up to help per users' better fathom the storyline(s) of the point. 


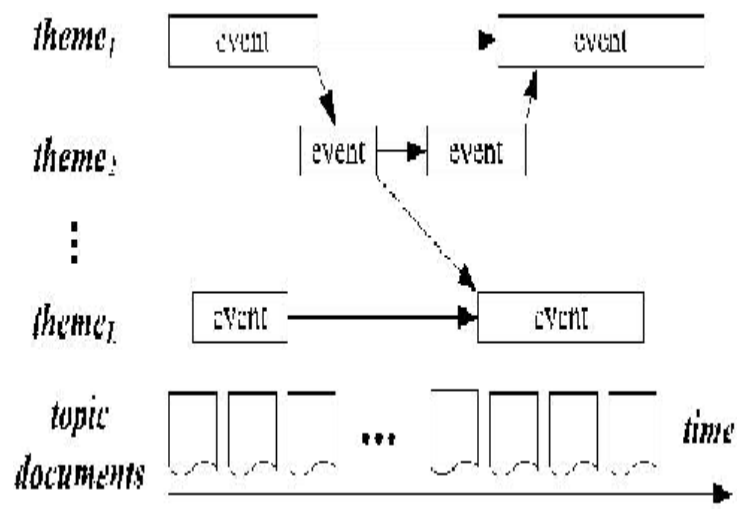

Fig. 3 Relationship between themes, events and event dependencies Design

This figure 3 outlines the connections between the subjects, occasions, and occasion conditions of a point in the proposed model.

\section{Theme Generation}

A grid A $1 / 4$ BT B, called a square affiliation grid, is a $n$ symmetric network in which the $(\mathrm{i}, \mathrm{j})$ - passage (signified as $\left.a_{i j}\right)$ is the internal result of sections I and $j$ in lattice B [10]. As a segment of $B$ is the term vector of a square, A speaks to the bury block affiliation. Henceforth, passages with an enormous worth infer a high connection between the relating pair of squares. A subject of a point is viewed as a totalled semantic profile of an assortment of squares, and can be spoken to as a vector $\mathrm{v}$ of measurement $\mathrm{n}$, where every passage indicates the level of connection of a square to the topic. Given the constitution of a vector v, vT Av registers the subject's relationship to the theme's substance.

\section{Event Segmentation and Summarization}

A subject $v j$ in VL is a standardized eigenvector of measurement $n$, where the $(i, j)$ - section vi; demonstrates the connection between a square I and a topic $\mathrm{j}$. As point blocks are recorded sequentially, a grouping of sections in vj with high qualities can be taken as an imperative occasion inserted in the subject, and valleys (i.e., a succession of little qualities) in vj might be occasion limits. Be that as it may, as per the meaning of eigenvectors, the indications of passages in an eigenvector are invertible.

In addition, Kleinberg and Nicholas and Dahlberg demonstrated that both the positive and negative passages of an eigenvector contain important semantics for depicting a specific idea installed in an archive corpus; and the sufficiency of a section decides the level of its relationship to the idea [9]. Note that the errands of our occasion division and discourse endpoint discovery are comparable in that the two of them attempt to recognize significant fragments of successive information. What's more, it is the sufficiency of successive information that decides the information's significance. There-front, we embrace Rabiner and Sambur's R-S endpoint discovery calculation utilized for occasion division. To portion occasions, the R-S calculation analyses the amplitude variety of an eigenvector to discover the endpoints that parcel the subject into a bunch of critical occasions.

\section{SIMULATION RESULTS}

The accompanying outcomes are acquired by the proposed framework where it got the Quality of data, forecast of right feelings. This methodology utilizes the Feature Extraction Technique with the TSCAN Scheme for forecast of the offered thoughts. While accomplishing work with this proposed framework we can acquire the earlier outcome which will overcome the current framework as far as anticipating the right feelings.

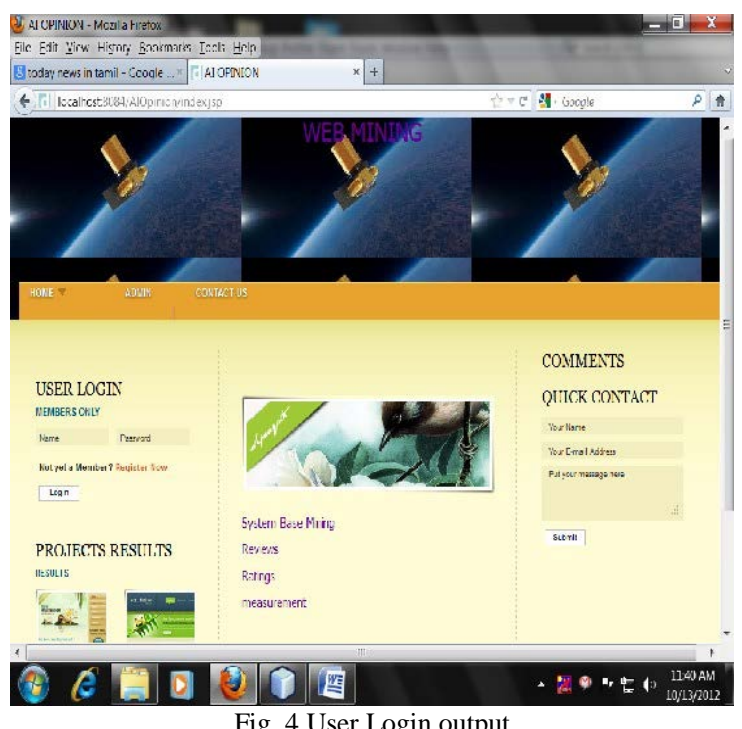

Fig. 4 User Login output

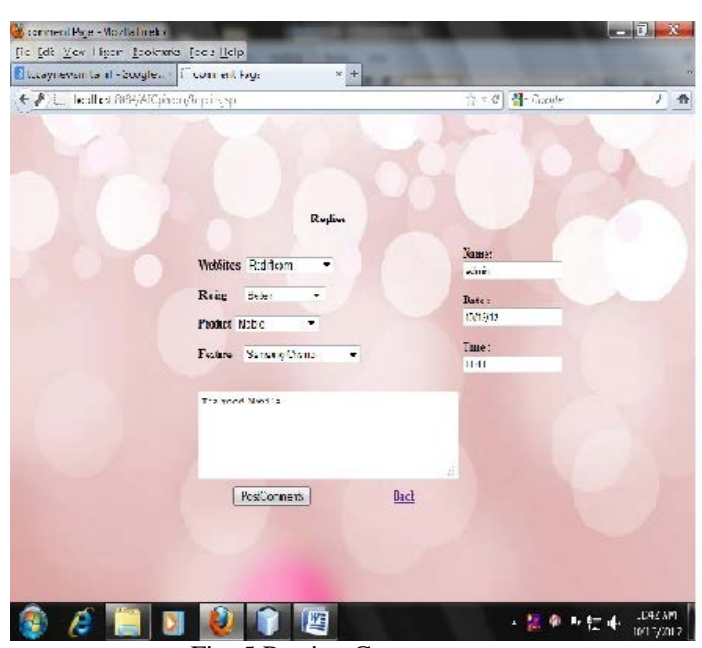

Fig. 5 Posting Comments output 


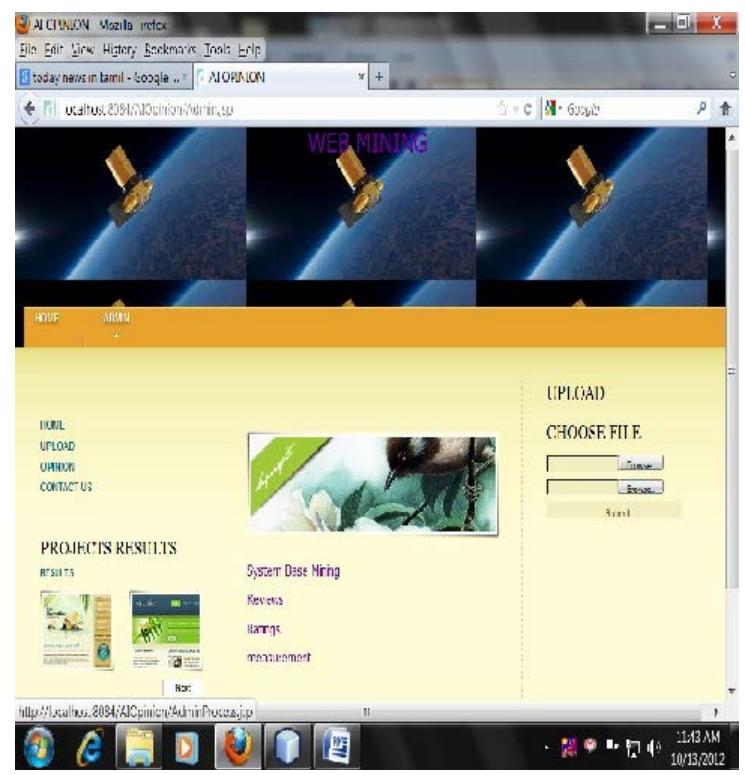

Fig. 6 Attempt to verify the Opinions output

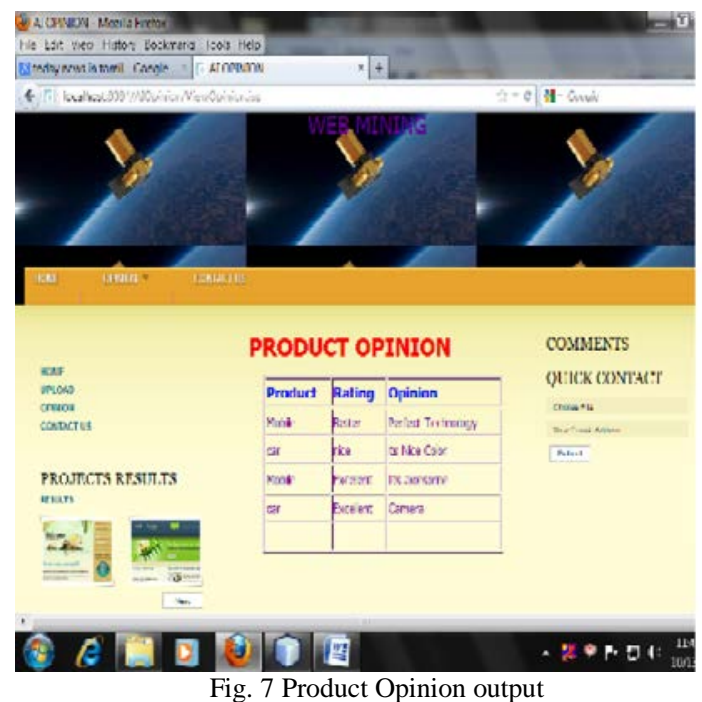

\section{PERFORMANCE EVALUATION}

Deciding the Threshold for Feature Combining I have utilized the 'accuracy' measure depicted as follows.

This accuracy measure assesses the proportion of effectively removed highlights by the framework. In exactness 'right component' demonstrates the element that corresponds with the physically labelled element under human watch for the analyses [9]. Albeit both 'exactness' and 'review' are significant assessing rules, we felt that 'exactness' is more suitable measure dependent on the perception that, in outline, it is more essential to give the right and accurate include data to the client than to give total data without missing highlights.

Accuracy $=$ No. of right highlights extricated by the situation No. of highlights separated by the framework
Review $=$ No. of right highlights removed by the situation No. of right highlights separated by the situation

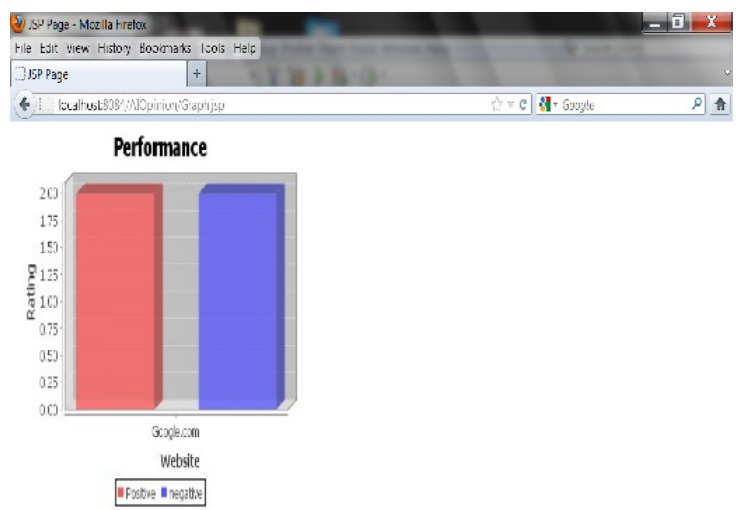

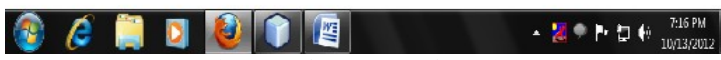

Fig.7 Performance of Product

It is investigated 300 client audit sentences for 'camera,' 'wireless,' and 'speaker' items to decide the edge for highlight blending. A trial is performed to get the accuracy proportions of highlight extraction by changing the edge esteems. As appeared in the accuracy is boosted when the limit is 0.1.Therefore we set the edge for highlight converging at 0.1 , which suggests that highlights with comparability esteems more noteworthy than 0.1 are combined.

\section{CONCLUSION}

Most outline techniques attempt to build the variety of synopses to cover all the significant data in the first records. In any case, when the reports to be summed up are identified with an advancing theme, outline techniques ought to likewise consider the fleeting properties of the subject to portray the advancement of storylines. In this paper, we have introduced a subject life structures framework called TSCAN, which concentrates topics, occasions, and occasion rundowns from theme records. In addition, the summed up occasions are related by their semantic and transient connections, and introduced graphically to frame a development diagram of the point. Investigations dependent on legitimate TDT4 themes show that TSCAN can deliver exceptionally agent rundowns that compare well to the reference synopses formed by specialists.

\section{REFERENCES}

[1] Minqing Hu and Bing Liu. "Mining and summarizing customer reviews", In: Proc. of the $10^{\text {th }}$ ACM S I G K D D -2016 international conference on knowledge discovery and data mining, Seattle, pp 168-177.

[2] N. Kobayashi, R. Iida, K. Inui andY. Matsumotto. "Opinion extraction using a learning-based anaphora resolution technique”, In 
Proc. of the second international joint conference on natural language processing (IJCNLP-04), Jeju Island, pp 173-178

[3] Wong TL, W. Lam "Learning to extract and summarize hot item features from multiple auction Web sites", Knowl Inf Syst, Vol.14, No.2, pp.143-160, 2008.

[4] E. Riloff., W. Janyce, and W. Theresa, "Learning Subjective Nouns Using Extraction Pattern Bootstrapping” In Proc.7th Conf. Natural Language Learning, pp 25-32, 2009.

[5] Yuanbin Wu, Qi Zhang, Xuanjing Huang and LideWu, "Phrase Dependency Parsing for Opinion Mining", Proceedings of the 2018 Conference on Empirical Methods in Natural Language Processing. Singapore, 6-7 August 2018, pp.1533-1541.

[6] Parma Nand, "On the use of Salience Weights in Anaphora Resolution”, In Proc. of NZCSRSC 2019, April 2008, Christchurch, New Zealand.
[7] Chih-Ping Wei, Yen-Ming Chen, Chin- Sheng Yang, Christopher and C. Yang. "Understanding what concerns consumers: a semantic approach to product feature extraction from consumer reviews", Springer-Verlag, 2019.

[8] G. Carenini and A. Pauls, "Multi- document Summarization of Evaluative Text”, In Proc. $11^{\text {th }}$ Conf. European Chapter of the ACL, 2019.

[9] M. Gamon., A. Aue., S. Corston-Oliver and E. Ringger, "Pulse: Mining Customer Opinions from Free Text", In Proc. $6^{\text {th }}$ Int. Symp. Advances in intelligent data analysis, pp121-132, 2005.

[10] J.Wiebe, "Learning Subjective Adjectives from Corpora”, In Proc. of $12^{\text {th }}$ Conference on Innovative Applications of Artificial Intelligence, 2019. 